\title{
An Electromicrographical Method for Evaluating Layer Structure of Polymeric Hollow Fiber Membrane
}

\author{
Kenji Kamide, Sakae Nakamura (née MiWa), Takaharu AKedo, \\ and Seiichi MANABE \\ Fundamental Research Laboratory for Fibers and Fiber-Forming Polymers, \\ Asahi Chemical Industry Co., Ltd., 11-7 Hacchounawate, \\ Takatsuki, Osaka 569, Japan
}

(Received September 14, 1988)

\begin{abstract}
An attempt was made to establish a novel electron micrographic method (aslant sliced thin sectional (ASTS) method) for evaluating pore characteristics, such as mean pore radius, pore radius distribution and porosity on inner layer at a given distance from the outer or inner wall of a porous polymeric hollow fiber membrane and to apply the method for clarifying the effect of preparatory conditions of the membrane on pore characteristics. Regenerated cellulose, cellulose diacetate, poly(vinyl alcohol) and polypropylene hollow fiber membranes for electron microscopic observation were sliced obliquely along the fiber axis at an angle $\theta\left(0<\theta<2.5^{\circ}\right)$ to give a thin section with a thickness less than $1 \mu \mathrm{m}$. The method affords us the information on continuous changes in pore characteristics along the radial direction, where the normal line of the section intercepts the fiber axis at angle 90- $\theta$ degree. The electron microscopic image obtained for a portion of an aslant sliced thin section could not be distinguished from those obtained by a thin section cut strictly parallel to the fiber axis (i.e., $\theta=0$ ). The ASTS method has the following advantages: (1) The location of a sliced section film can be evaluated accurately; (2) only a single operation to slice the sample section is needed; and (3) information on pore characteristics can be obtained continuously along the membrane thickness.
\end{abstract}

KEY WORDS Pore Characteristic/Micro-Phase Separation / Hollow Fiber / Electron Microscope / Porous Polymeric Membrane / Ultra-Thin Section / Mean Pore Radius / Regenerated Cellulose / Layer Structure /

Various methods for evaluating mean pore radius and pore radius distribution of a porous polymeric membrane have been hitherto proposed, such as the electron microscopic (EM) method and water filtration flow rate method. ${ }^{1}$ These conventional methods can be applied to evaluate the pore characteristics of not only plane membranes but also hollow fiber membranes, if the pore characteristics do not change significantly according to their location in the direction of membrane thickness. However, at least for plane membranes, it was proved that pore characteristics change depending on the location in the thickness direction. Merin and Cheryan ${ }^{2}$ studied the structure of a plane ultrafiltration polysulfone mem- brane, using scanning and transmission electron microscopes (SEM and TEM) and estimated pore radius distribution of the surface layer of the membrane. Based on electron microscopic observation on a cross section of a cellulose acetate plane membrane, Purz et al. ${ }^{3}$ suggested that pore radius distribution depends on the distance from the surface of the membrane. However, in characterizing a membrane by electron microscope, only the observation of the surface and cross section of a membrane has been carried out to date. ${ }^{2-4}$

An information on change in pore characteristics in the thickness direction is prerequisite to estimation of the pore characteristics of porous polymeric membranes. This is par- 
ticularly important for membranes prepared by micro-phase separation, since change of pore characteristics in the thickness direction is predicted theoretically. ${ }^{5}$

For a plane membrane, applying the EM method to a series of thin section cut in parallel to the membrane surface, Manabe et $a l^{6}$ experimentally verified the change in pore characteristics in the thickness direction and found it correlated with change in phase separation conditions with the thickness direction of membrane.

Although hollow fiber porous membranes are more readily applied in industry than plane membranes, characterization science and technology of pore characteristics of hollow fiber membrane remains at a very primitive stage. As far as a hollow fiber membrane is concerned, no study has reported on multi-layer structure and no conventional methods for analyzing the layer structure useful for plane membrane can be applied to hollow fiber membranes. For example, the thin sectional method proposed by Manabe et $a l^{6}{ }^{6}$ needs great skill in experiments in applying it to hollow fiber membranes.

In this paper, we propose a new method for evaluating pore characteristics as a function of the distance from the membrane surface by slicing off a thin section film from a hollow fiber and analyzing the thin section film and apply this method to various commercially available hollow fiber membranes.

\section{EXPERIMENTAL}

\section{Membrane Samples}

Two porous regenerated cellulose hollow fiber membranes, sample codes $\mathrm{RCa}$ and $\mathrm{RCb}$, whose mean pore diameters are $40.1 \mathrm{~nm}$ and $22.1 \mathrm{~nm}$, respectively, as determined by the water filtration flow rate method were prepared in our laboratory by applying the microphase separation method to cellulose cuprammonium solutions. ${ }^{6}$ Some commercially available cellulose diacetate (CDA), poly(vinyl alcohol) (PVA), and polypropylene (PP) hollow fiber membranes, listed in Table I, were also used.

\section{Electron Microscopic Observation}

A single hollow fiber was embedded in a block of acrylic resin (3/7 (weight ratio) mixture of $n$-butyl acrylate/methyl methacrylate) and sliced using Ultratome type 8800 (manufactured by LKB Co., Ltd., Sweden) to give a thin film (i.e., section) with a thickness less than $1 \mu \mathrm{m}$, whose normal line intercepted the fiber axis at angle 90- $\theta$ degree (see Figure 1). Here $\theta$ ranged from 0 to $90^{\circ}$. The case of $\theta=0$ is the case where the thin section is parallel to the fiber axis and in this case $(\theta=0)$ five sections were sliced at different distances $Z$ from the inner wall. The acrylic resin remaining in the section was dissolved and removed by dropping chloroform on the section. This section was coated with $\mathrm{Au}$ of $20 \mathrm{~nm}$ thick using ION SPUTTER JFC-1100 (manufactured by JOEL Co., Ltd., Japan) and observed under SEM

Table I. Commercially available hollow fiber membranes used in this study

\begin{tabular}{|c|c|c|c|c|}
\hline Materials & Manufacturer & Trade name & $\begin{array}{c}\text { Membrane } \\
\text { thickness } / \mu \mathrm{m}\end{array}$ & $\begin{array}{c}\text { Preparation } \\
\text { Method }\end{array}$ \\
\hline Cellulose diacetate & Asahi Medical Co., Ltd. & $\begin{array}{c}\text { Plasmaflow } \\
\text { AP-05H }\end{array}$ & 53 & $\begin{array}{r}\text { Micro-phase } \\
\text { separation }\end{array}$ \\
\hline Poly(vinyl alcohol) & Kuraray Co., Ltd. & Plasmacure & 65 & $\begin{array}{l}\text { Solvet } \\
\quad \text { extraction }\end{array}$ \\
\hline Polypropylene & Mitsubishi Rayon Co., Ltd. & Diacrystal & 27 & $\begin{array}{l}\text { Drawing and } \\
\text { anealing }\end{array}$ \\
\hline
\end{tabular}




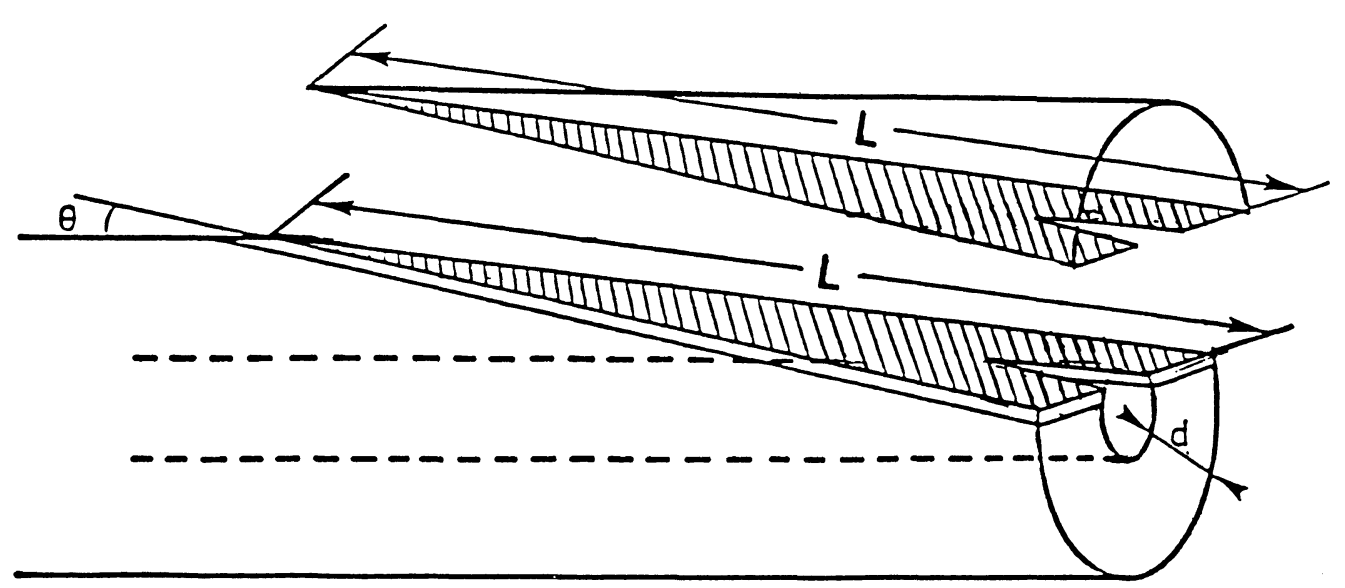

Figure 1. Schematic representation of a sliced hollow fiber membrane, whose normal line intercepts with the fiber axis at angle $90-\theta$ degree.

(JSM 35CF, manufactured by JOEL Co., Ltd., Japan).

$\theta$ was calculated from the equation:

$$
\theta=\sin ^{-1}(d / L)
$$

where $d$ is the membrane thickness and $\mathrm{L}$ is the total length of the section cut from hollow fiber, as shown in Figure 2. The distance $Z$ was calculated from $\theta$ and the distance $x$ from the inner wall edge in the section through the simple relation:

$$
Z=x \sin \theta
$$

Applying the stereological method proposed by Manabe et al., ${ }^{7}$ the porosity Pre, the $i$-th mean pore radius $\bar{r}_{i}$ and the pore radius distribution function for a small portion in the section were evaluated as a function of $Z / d$, (the distance from the inner wall)/(membrane thickness).

\section{RESULTS AND DISCUSSION}

Maximum $\theta$ Value Determined with Reasonable Accuracy in the Aslant Sliced Thin Sectional Method

When $\theta=0$, there is a drawback besides the necessity for skilled techniques in analysis of pore characteristics of hollow fiber membrane.

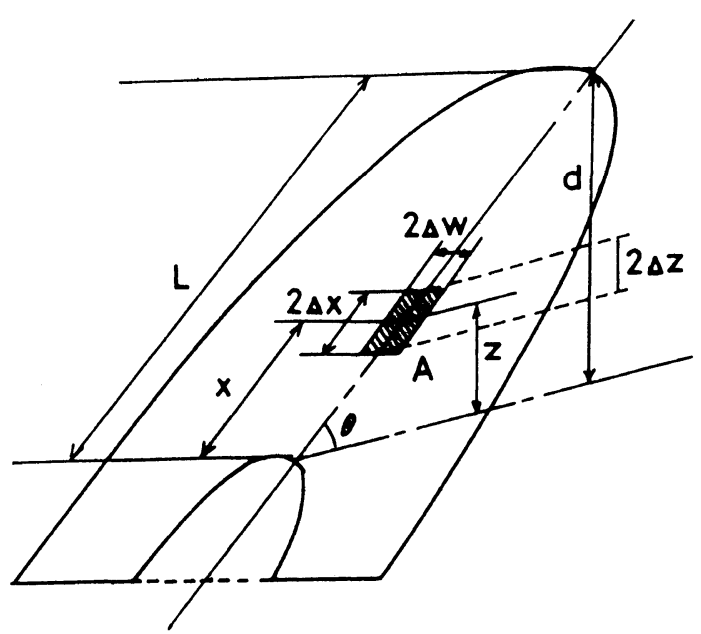

Figure 2. Schematic representation of a sliced thin section of hollow fiber membrane, whose normal line intercepts with the fiber axis at angle 90- $\theta$ degree: $L$, length of a thin section; $d$, thickness of hollow fiber membrane; $x$, length from inner surface to the center of hatched area $\mathrm{A}$ in the section; $z$, distance between $\mathrm{A}$ and inner surface of hollow fiber membrane $(=x \sin \theta) ; \Delta x$, $\Delta z$, length and width of an electron micrograph for analysis of pore characteristics, respectively.

Only discrete data of pore characteristics regarding the distance from the surface of membrane are obtained. For $\theta \neq 0$, these experimental drawbacks mentioned, encountered in the case of $\theta=0$, vanish, although inaccuracy of $Z$ in quantitative analysis of electron micro- 

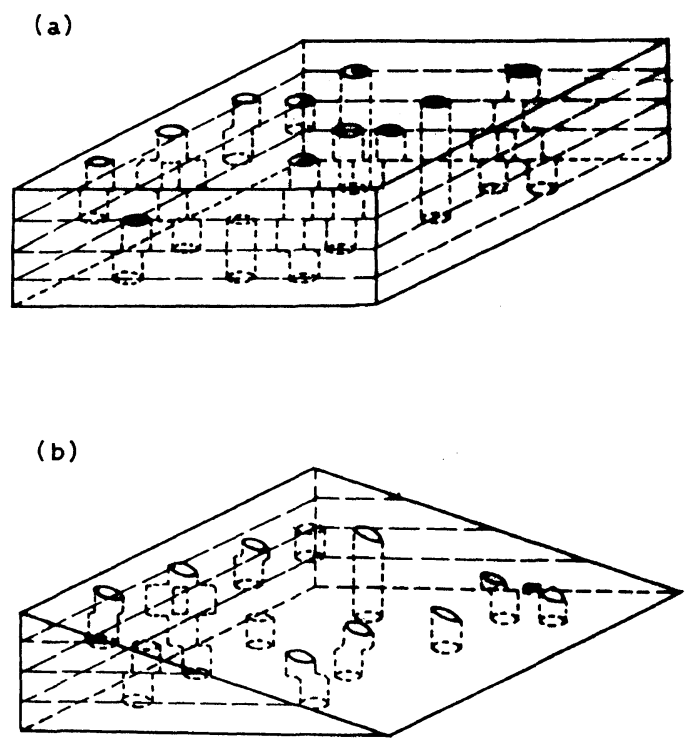

Figure 3. Schematic representation of membrane with layer structure, which has straight-through cylindrical pores in all layers: (a), membrane composed of 4 layers; (b), membrane cut off obliquely to membrane surface.

graphs using stereology still exists. But when $\theta$ increases, the shape of a pore in the thin section may be distorted apparently from that in the thin section at $\theta=0$. Thus, there may exist a maximum limit of $\theta$, which gives accurate pore characteristics within experimental uncertainty.

The degree of distortion of apparent pore shape observed for a sliced section with $\theta \neq 90^{\circ}$ can be theoretically calculated for a model membrane which has typical pore shapes such as circular straight-through cylindrical pores in all layers constituting the membrane, as shown in Figure 3. The distortion of pore shape from a circle, as observed for the sliced section, can be represented by the difference between the maximum cut off length $2 r_{\mathrm{m}}$ and the diameter $2 r$ of the circle. Figure 4 shows the dependence of $\left(r_{\mathrm{m}}-r\right) / r$ on the sliced angle $\theta$. As expected, $\left(r_{\mathrm{m}}-r\right) / r$ increases with $\theta$, being less than 0.01 . Therefore, we can conclude that the distortion of pore shape by slicing at $\theta \neq 0$ is negligibly small, in the range $\theta<8^{\circ}$.

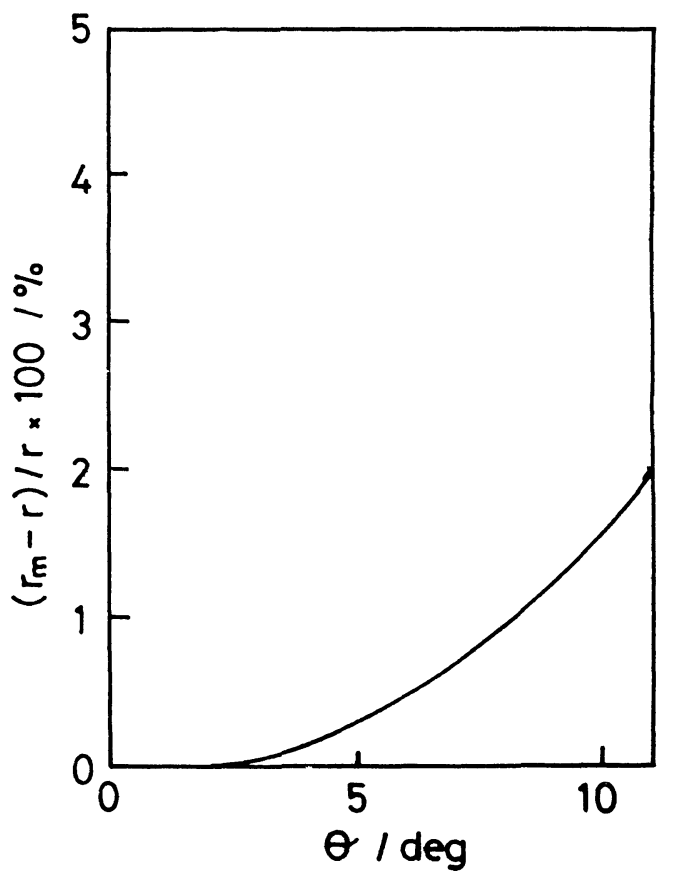

Figure 4. $\theta$ dependence of $\left(r_{\mathrm{m}}-r\right) / r: 2 r_{\mathrm{m}}$; maximum cut off length of straight-through cylindrical pore with radius $r$ in a section sliced obliquely at angle $\theta$.

Figure 5a shows an example of an electron micrograph of a RCa membrane at $Z / d=0.48$, where the black part is a pore and the hatched parts are regarded as pores, as schematically shown in Figure 5b. In order to analyze an electron micrograph of the membrane quantitatively with the help of stereology, the area of the photograph to be analyzed must contain more than 200 pores, generally. For this purpose, the size of $10 \mu \mathrm{m} \times 10 \mu \mathrm{m}$ is practically sufficient for microfiltration (MF) membrane. Figure 6 shows $F(x)$ vs. the $x$ curve given by analyzing the electron micrograph in Figure 5, where $F(x)$ is the number of pores which intercept test lines of length $x \Delta D \sim(x+1) \Delta D$. Here $\Delta D$ is defined as $\Delta D=D_{\max } / I$, where $D_{\max }$ is the maximum cut off length of pores by test lines and $I$ is determined based on the accuracy of $F(x)$ to be calculated. In Figure 6, a smooth curve of $F(x)$ was obtained, indicating that the size of the portion of the section is sufficient 


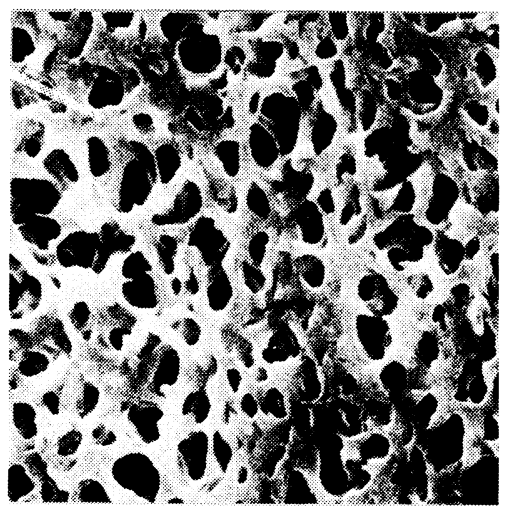

(a)

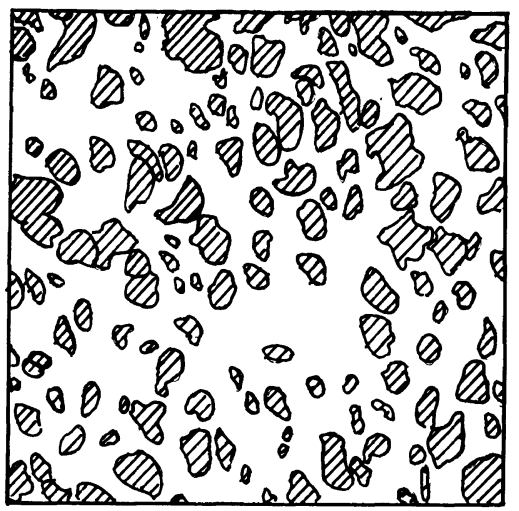

(b)

Figure 5. Electron micrograph for analysis of pore radius distribution: (a), electron micrograph of $\mathrm{RCa}$ membrane at $Z / d=0.48$; (b), schematic representation of Figure 5a, hatched parts are regarded as pores.

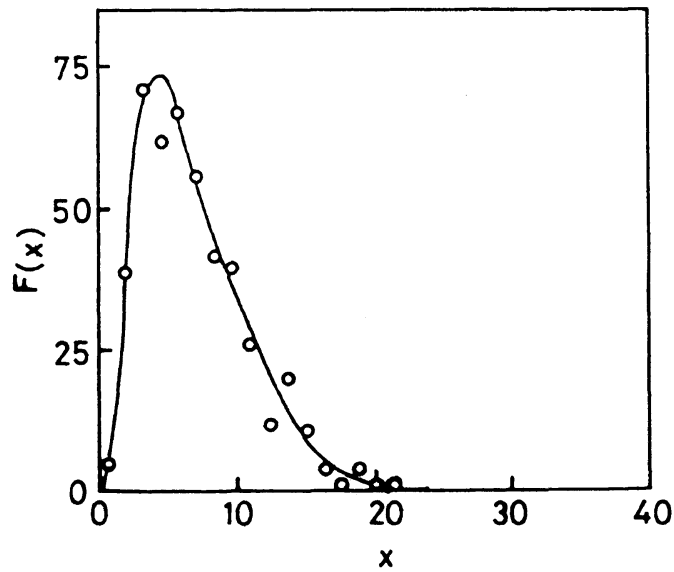

Figure 6. Relation between $F(x)$ and $x: F(x)$, distribution function of the length $x \Delta D$ of test line cut off by pores in electron micrograph.

for analysis of pore radius distribution. However, in analyzing an electron micrograph, there may exist error $\Delta Z$ in calculating $Z$, due to averaging $Z$ of the portion of the section in the electron micragraph with the length $2 \Delta X$ and width $2 \Delta W$ (see Figure 2).

As described before, $10 \mu \mathrm{m}$ is practically approved value for $2 \Delta X$ or $2 \Delta W$, and eq 2 is rewrited as

$$
\Delta Z / d=\Delta X \sin \theta / d
$$

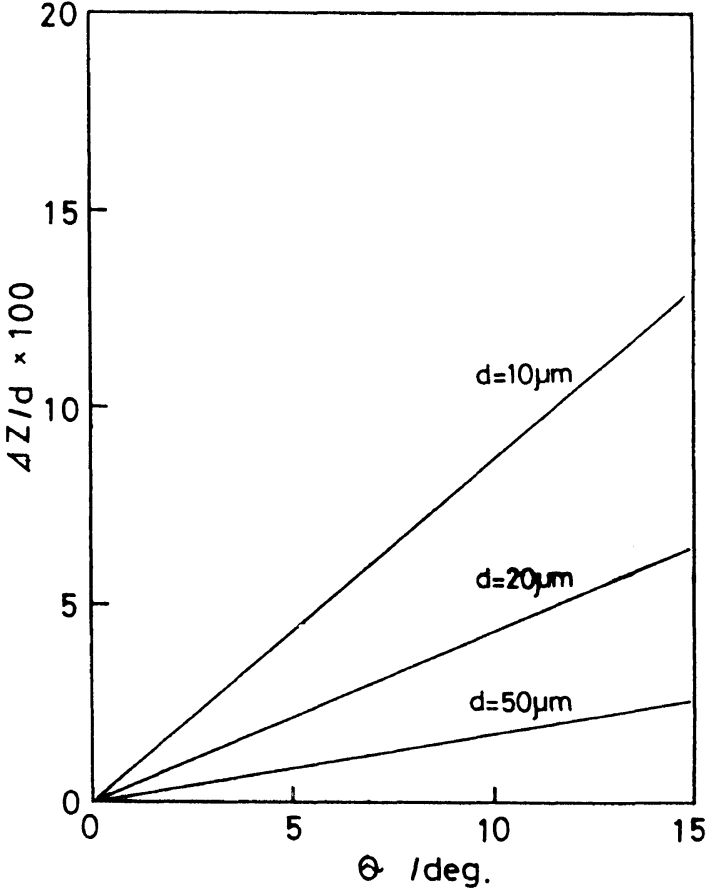

Figure 7. $\theta$ dependence of $\Delta Z / d$ at various $d$ values, in the case of $\Delta x=5 \mu \mathrm{m}$ in eq 2 .

Figure 7 is an example of the dependence of $\Delta Z / d$ on $\theta$ for a given $d$ value when $\Delta X=5 \mu \mathrm{m}$. For a practical MF membrane, $d \geqq 20 \mu \mathrm{m}$ and $\Delta X=5 \mu \mathrm{m}$ as mentioned before, then $\theta$ should 


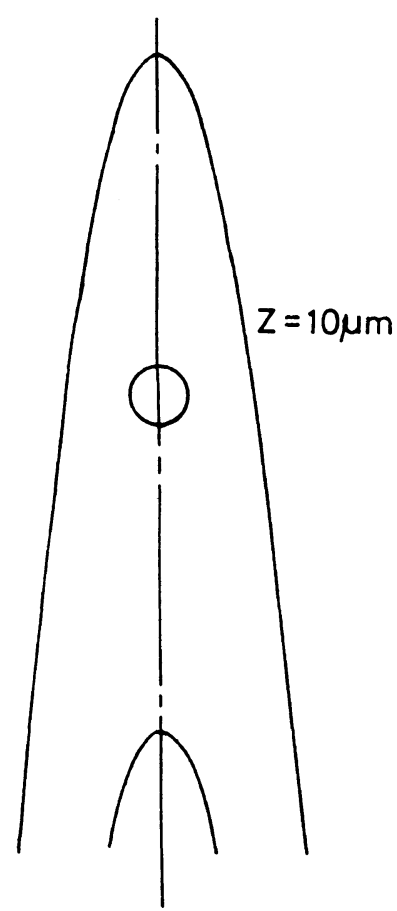

(a)

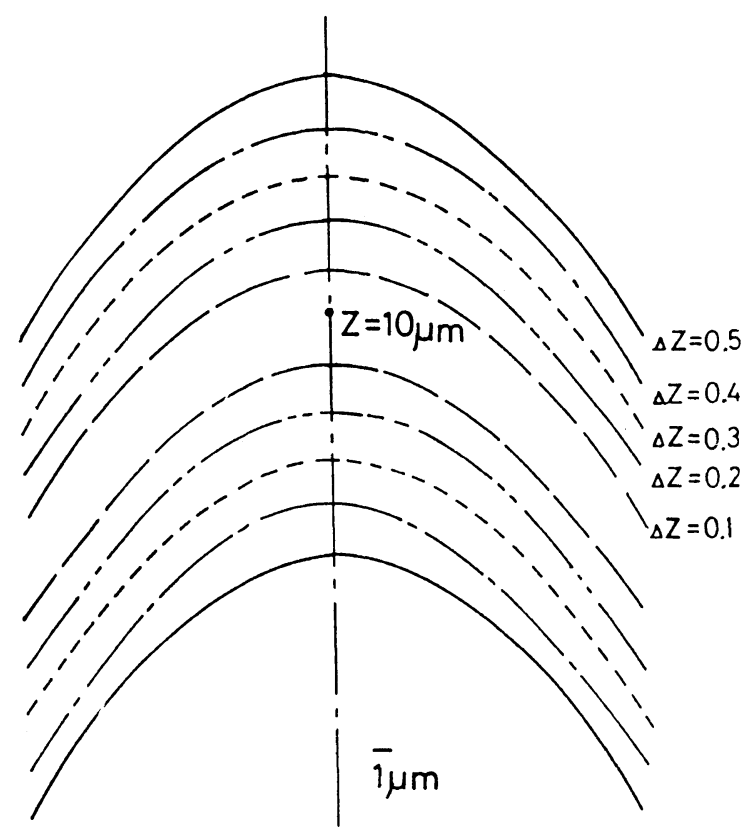

(b)

Figure 8. Schematic representation of thin section of hollow fiber membrane with $d=20 \mu \mathrm{m}$ and inner diameter $200 \mu \mathrm{m}$, given under $\theta=2^{\circ}$ (a) and expanded figure of a circle in Figure 8(a): The curves in Figure 8 (b) indicate the identical value of $\Delta z=0.1,0.2,0.3,0.4$, and 0.5 , respectively. In the area enclosed with two curves having the same identical value of $\Delta z$, the error of $Z$ is within $\Delta Z$.

be less than $2.3^{\circ}$.

Figure 8 illustrates a portion area in a sliced thin section obtained when the inner diameter of a hollow fiber membrane is $300 \mu \mathrm{m}, d=$ $20 \mu \mathrm{m}$, and $\Delta Z / d=0.005,0.01,0.02,0.03$, and 0.04 , respectively. Because $\Delta Z / d<0.01$ may be accepted in comparison with an experimental inaccuracy, the area in Figure 8 for $\Delta Z / d<0.01$ can be used and this is larger than $10 \mu \mathrm{m} \times 10 \mu \mathrm{m}$. Thus, the maximum $\theta$ value $\left(\theta_{\mathrm{m}}\right)$ is $\sin ^{-1}(\Delta X / d)$. This method for evaluating pore characteristics is hereafter simply named the "aslant sliced thin sectional (ASTS)" method.

In order to ensure experimentally the reliability of the considerations mentioned above, a thin section with $1 \mu \mathrm{m}$ thickness was prepared with an angle of $\theta=1.1^{\circ}, 3.5^{\circ}$, and $4.4^{\circ}$ for $\mathrm{RCb}$ membrane. The mean pore diameters $2\left(\bar{r}_{3} \bar{r}_{4}\right)^{1 / 2}$ where $\bar{r}_{3}$ and $\bar{r}_{4}$ are the 3 rd and 4 th moments of pore radius defined by Kamide and Manabe ${ }^{1}$ before, and the porosity Pre were evaluated from $\mathrm{RCb}$ membrane's electron micrographs and plotted in Figure 9 as a function of $Z / d$. For an $\mathrm{RCb}$ membrane, $d=20 \mu \mathrm{m}$ and $\Delta X>10 \mu \mathrm{m}$, and thus $\theta_{\mathrm{m}}=$ $2.34^{\circ}$. Interestingly, for $\theta=1.1^{\circ}$ there are two minima in $2\left(\bar{r}_{3} \bar{r}_{4}\right)^{1 / 2}$ and one maximum in Pre. When $\theta>\theta_{\mathrm{m}}$, detailed information on $Z / d$ dependence of $2\left(\bar{r}_{3} \bar{r}_{4}\right)^{1 / 2}$ cannot be obtained but only a smooth curve without a maximum.

Figure 10 shows electron micrographs of some portions of RCa membrane, cut off at $\theta=1.5^{\circ}$ and $\theta=0$, respectively. Figure 11 shows $2\left(\bar{r}_{3} \bar{r}_{4}\right)^{1 / 2}$, evaluated from Figure 10 , as a function of $Z / d$. In the case of $\theta=1.5^{\circ}$ (Figure 


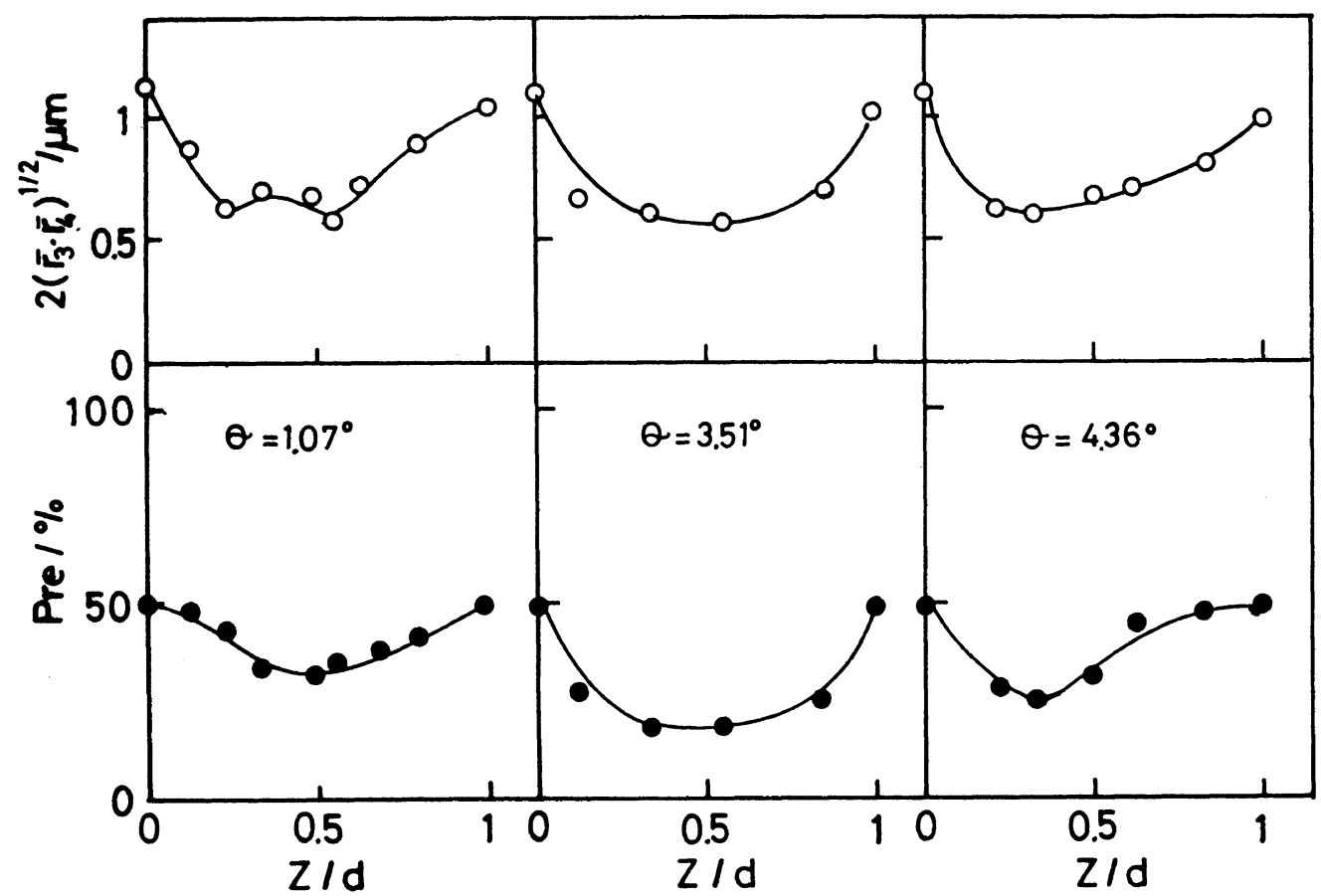

Figure 9. $\mathbf{Z} / d$ dependence of mean pore diameter $2\left(\bar{r}_{3} \bar{r}_{4}\right)^{1 / 2}$ and porosity Pre of RCb membrane, at various slicing angle $\theta$.

(a)

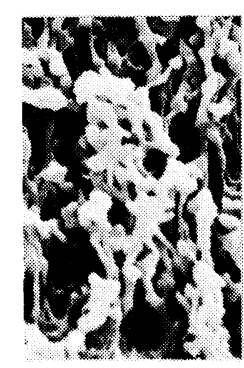

(1)

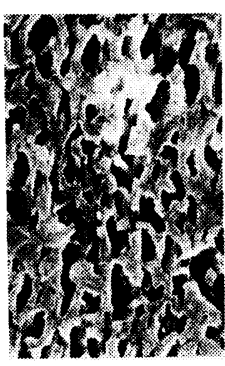

(2)

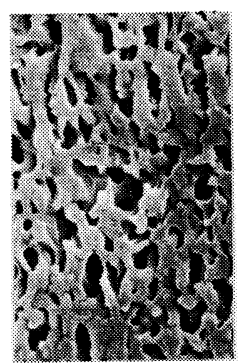

(2)

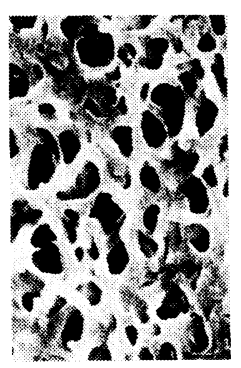

(3)

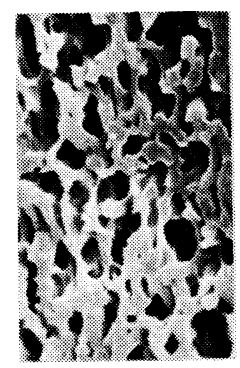

(3)

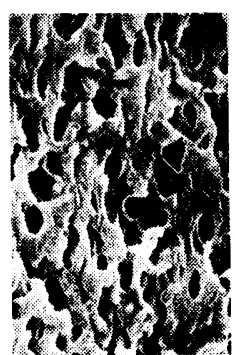

(4)

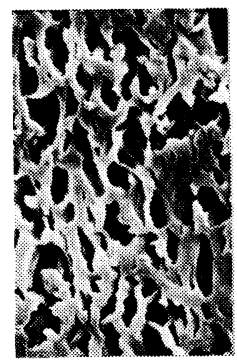

(4)

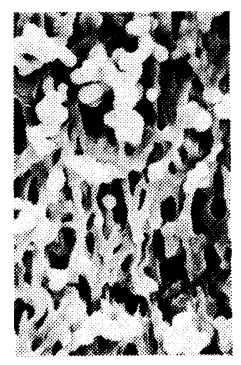

(5)

(b)

Figure 10. Electron micrographs of RCa membrane observed at various $Z / d$ : (a), $\theta=0$; (b), $\theta=1.5^{\circ}$. (1), inner surface; (2), $Z / d=0.167$; (3), $Z / d=0.48$; (4), $Z / d=0.944$; (5), outer surface. 


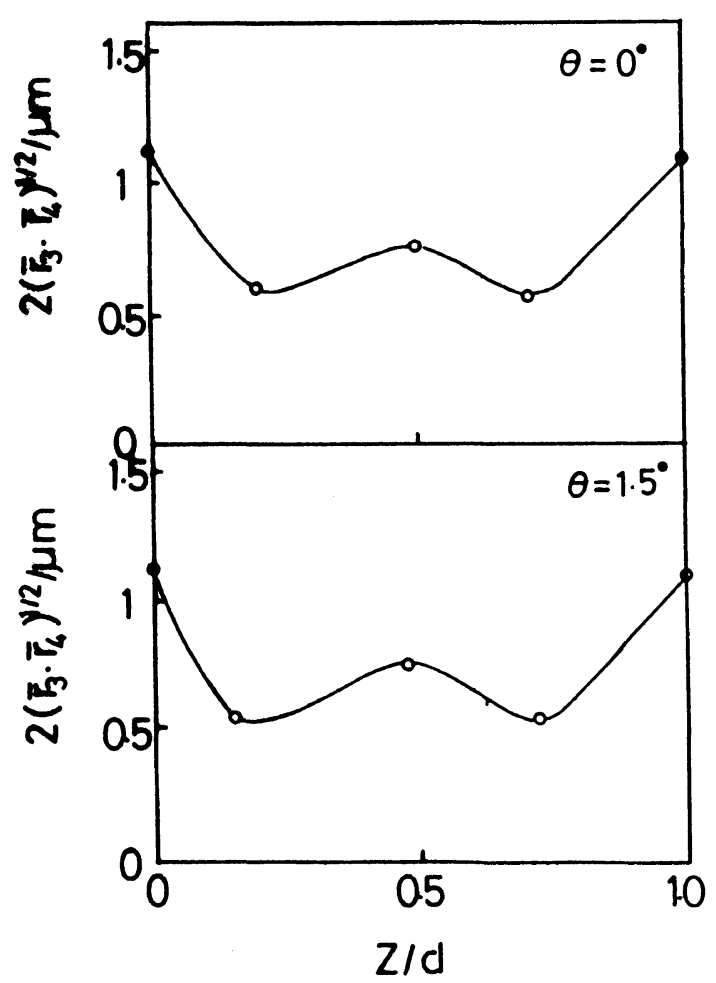

Figure 11. $Z / d$ dependence of pore diameter for the sections of $\theta=1.5^{\circ}$ (a) and $\theta=0$ (b) of RCa hollow fiber membrane.

10b), continous $2\left(\bar{r}_{3} \bar{r}_{4}\right)^{1 / 2}$ values for $Z / d$ can be obtained by the ASTS method, but only five $2\left(\bar{r}_{3} \bar{r}_{4}\right)^{1 / 2}$ values corresponding to five $Z / d$ could be calculated for $\theta=0$. Both electron microscopic images in Figures 10a and 10b are similar to each other and thus difficult to be distinguished. The $Z / d$ dependence of pore characteristics is the same in both cases.

As predicted above, for $\theta<\theta_{\mathrm{m}}$, the pore characteristics coincide with those of $\theta=0$ and for $\theta>\theta_{\mathrm{m}}$, the pore characteristics deviate apparently from those of $\theta=0$ (see Figures 9 and 11).

Accordingly, the ASTS method applied to hollow fiber membrane gives accurate and detailed structural images of pore characteristics in the thickness direction. However, for a given $Z$, only one pore radius distribution is obtained and fluctuation of pore radius distri- bution in a layer at $\mathbf{Z}=$ constant cannot be obtained. To examine the fluctuation of pore radius distribution in a given layer, it is necessary to slice several thin sections of hollow fiber membrane. Neverthless, the ASTS method is useful for characterizing hollow fiber membranes.

Characteristic Features of a Porous Hollow Fiber Prepared by the Micro-Phase Separation Method

Figures 12, 13, and 14 show scanning electron micrographs at various $Z / d$ values for CDA, PVA, and PP hollow fiber membranes. The change in the mean pore diameter $2\left(\bar{r}_{3} \bar{r}_{4}\right)^{1 / 2}$ with $Z / d$ of RC, CDA, PVA, and PP hollow fibers can be schematically represented in Figure 15. Except for the RC hollow fiber, the mean pore size of either the inner or outer wall surface is smaller than that of the inside part of the membrane. In the inside of the membrane of PVA and PP hollow fibers, variation in the mean pore size with $Z / \mathrm{d}$ is relatively small compared with that of $R C$ and CDA membranes. Of course dependence of the mean pore size on $Z / d$ varies depending on the mechanism by which membranes are formed, in other words, phenomenologically on membrane preparing conditions.

The mean pore size $v s$. $Z / d$ for $\mathrm{RC}$ hollow fiber shows two minima and one maximum in Figure 10. The pore shape also changes with $Z / d$ : When $Z / d$ is less than 0.35 and more than 0.65 , the pore shape is uncircular. The pore wall is constructed of secondary particles. These particles are characteristic features of a membrane prepared from a solution in which the cellulose concentration at the phase separation is less than the critical solution concentration.

Figure 16 shows a scanning electron micrograph of a RC hollow fiber with $Z / d=0.40$. This photograph is simillar to the typical pattern obtained by computer simulation of the spinodal decomposition. ${ }^{8}$ When $Z / d$ ranges from 0.45 to 0.55 , the circular pores 


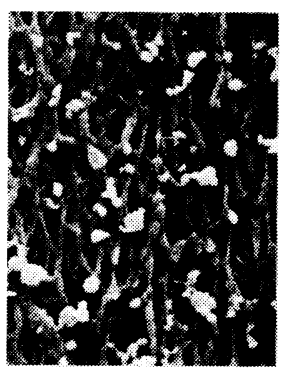

(1)

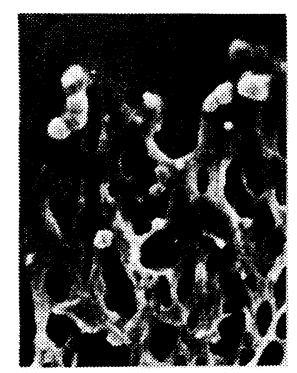

(2)

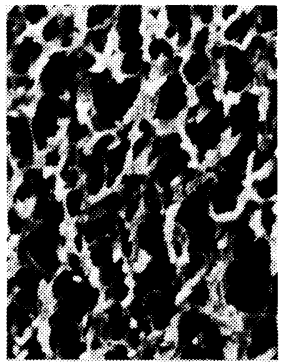

(5)

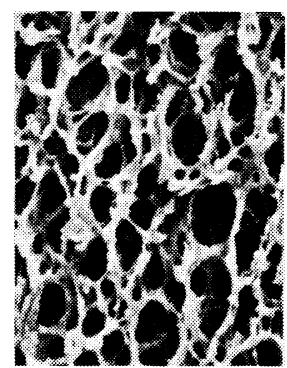

(3)

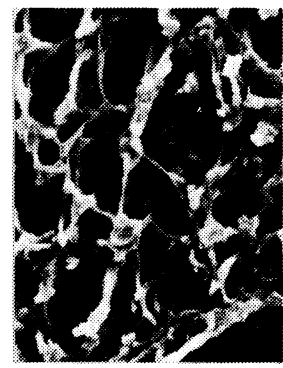

(6)

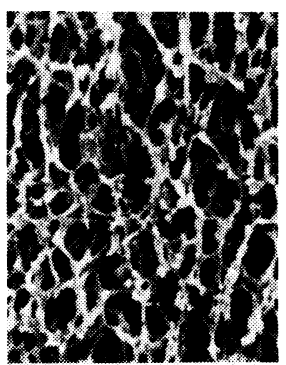

(4)

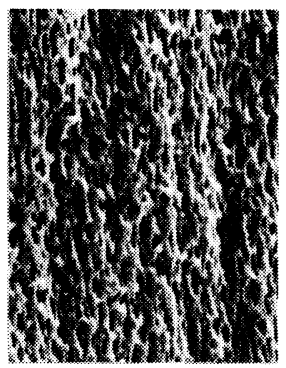

(7)

Figure 12. Electron micrographs of CDA membrane observed at various $Z / d$ at $\theta=1.0^{\circ}$ : (1), inner surface; (2), $Z / d=0.019 ;$ (3), $Z / d=0.28$; (4), $Z / d=0.47$; (5), $Z / d=0.94 ;$ (6), $Z / d=0.98$; (7), outer surface.

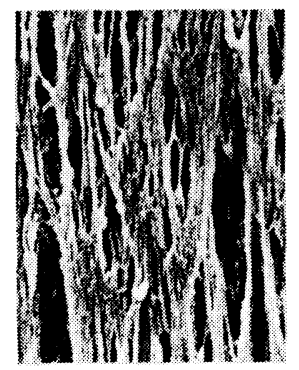

(1)

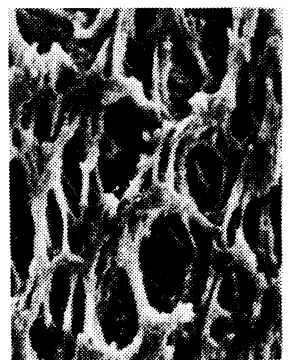

(2)

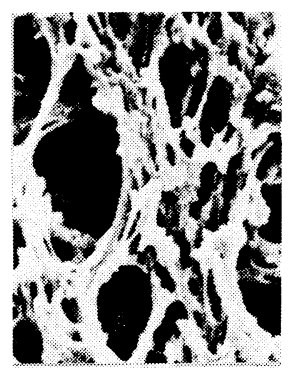

(5)

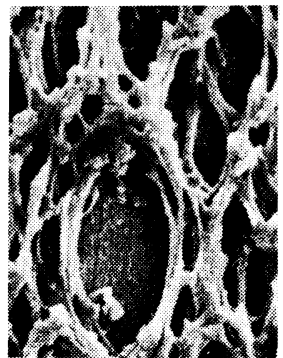

(3)

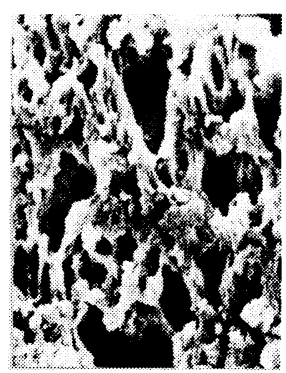

(6)

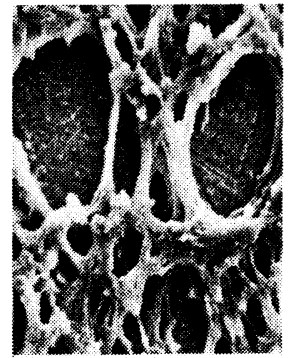

(4)

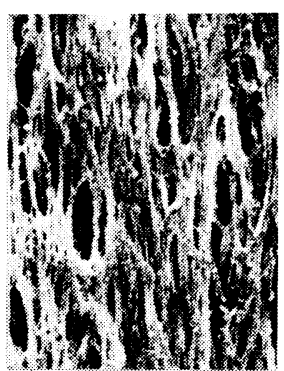

(7) $\overline{1 \mu m}$

Figure 13. Electron micrographs of PVA membrane observed at various $Z / d$ at $\theta=1.0^{\circ}$ : (1), inner surface; (2), $Z / d=0.013 ;$ (3), $Z / d=0.13 ;$ (4), $Z / d=0.53 ;(5), Z / d=0.62 ;(6), Z / d=0.99 ;$ (7), outer surface. 


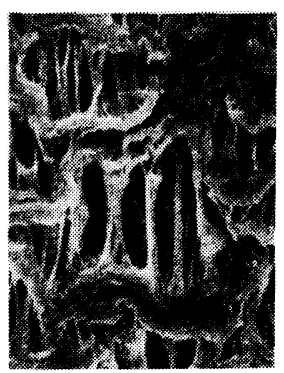

(1)

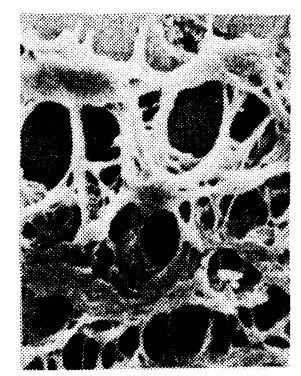

(2)

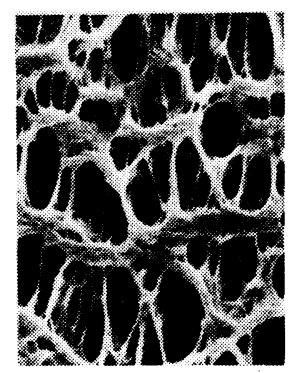

(5)

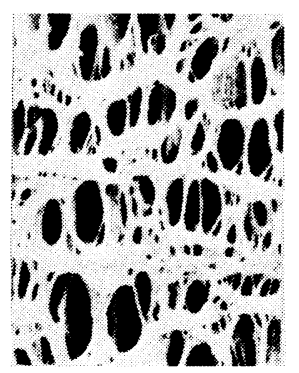

(3)

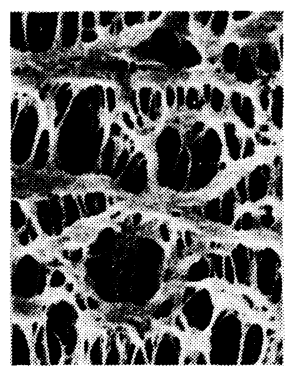

(6)

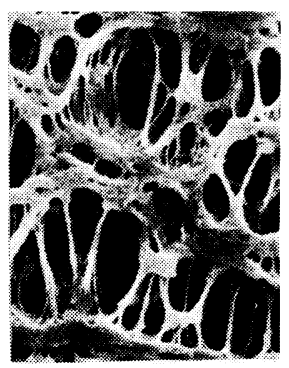

(4)

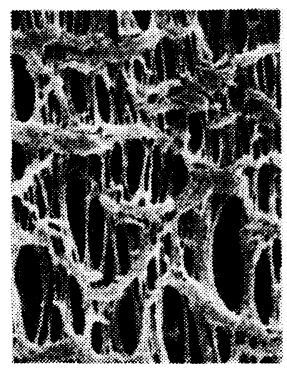

(7) $\overline{1 \mu m}$

Figure 14. Electron micrographs of PP membrane observed at various $Z / d$ at $\theta=1.0^{\circ}:(1)$, inner surface; (2), $Z / d=0.016$, (3), $Z / d=0.078$; (4), $Z / d=0.50$; (5), $Z / d=0.67$; (6), $Z / d=0.99$; (7), outer surface.

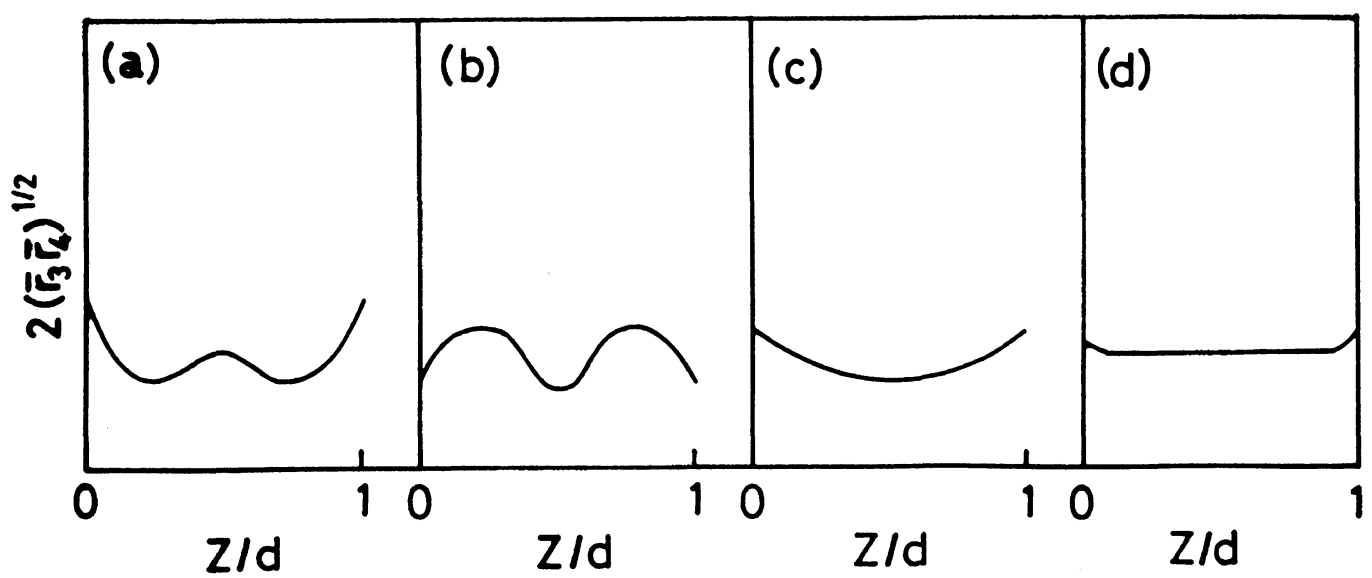

Figure 15. Schematic representation of $Z / d$ dependence of mean pore diameter $2\left(\bar{r}_{3} \bar{r}_{4}\right)^{1 / 2}$ for various hollow fiber membrane: (a), RC; (b), CDA; (c), PVA; (d), PP.

characteristic of the appearance of a membrane prepared from solution with a polymer concentration higher than the critical concentration, were observed. Thus, significant change may occur in polymer concentration with $Z / d$ in the process of the micro-phase separation. This is partly supported by the experimental fact that the porosity Pre has one minimum for a RCa hollow fiver, as shown in Figure 9.

The RC hollow fiber was prepared using a ternary coagulant mixture of acetone, water 


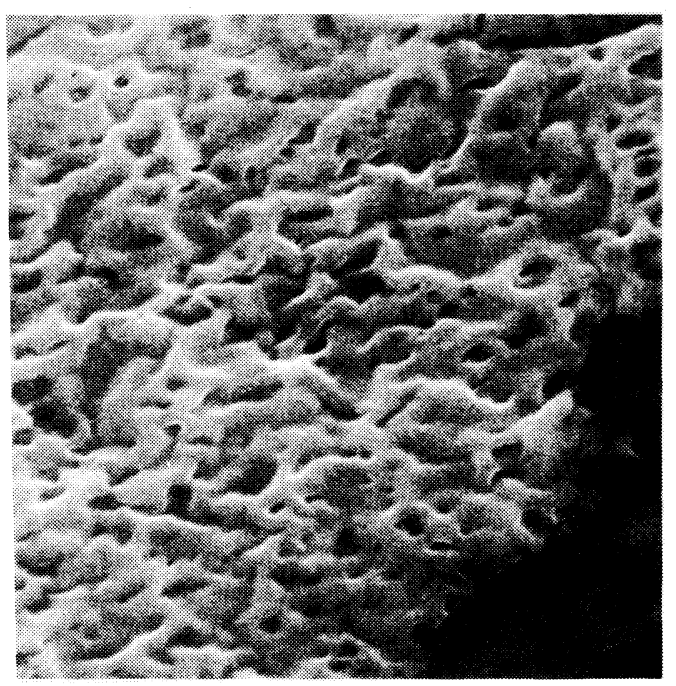

(a)

$0.5 \mu \mathrm{m}$

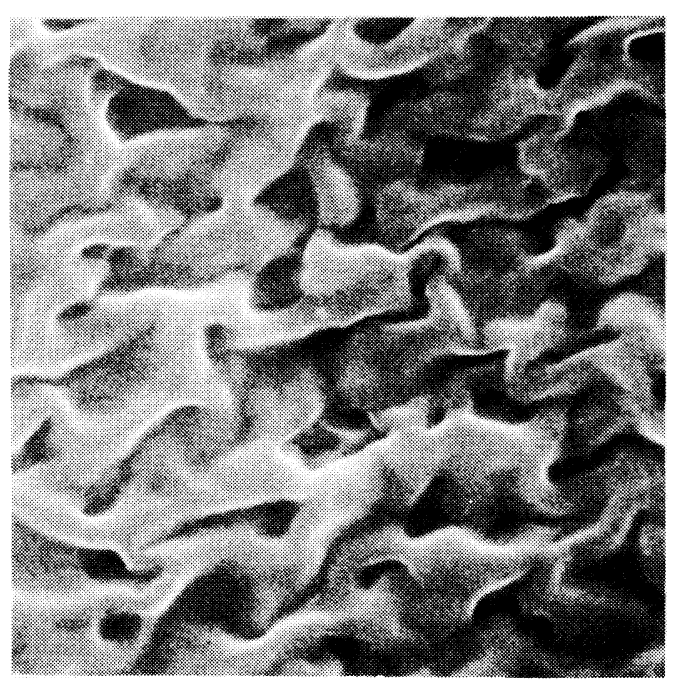

(b)

$0.5 \mu \mathrm{m}$

Figure 16. Electron micrographs of RCa membrane at $Z / d=0.4$ at $\theta=1.5^{\circ}$.

and ammonia, from a cellulose cuprammonium solution with a cellulose concentration less than the critical concentration. ${ }^{9}$
The micro-phase separation may occur simultaneously from the outer and inner surfaces of the solution resulting in the same pore characteristics in both membrane surfaces. When the micro-phase separation proceeds at the same time from outer and inner surfaces with the same speed to the inside of the membrane, as is the case of a RC hollow fiber, the pore characteristics $v s . Z / d$ curves may show a symmetrical pattern with respect to $Z / d=0.5$. The increase in cellulose concentration may occur at the inside of the membrane in the case of a RC hollow fiber, as was indirectly shown by Manabe et al. ${ }^{6}$ for RC plane type membranes. The feed solution contacts the coagulant at both sides of the inner and outer surfaces. Consequently, the cellulose concentration at the time of micro-phase separation at the central position $(Z / d$ is between 0.45 and 0.55 in the case of RC hollow fiber) may exceed the critical concentration, since the pore shape at the central position is circular. In contrast, the change in pore characteristics with $Z / d$ gives information on compositional changes with $Z / d$ during spining.

The CDA hollow fiber membrance (by Asahi Medical Co.) was prepared by the micro-phase separation method. The spinning conditions were different from those of the RC membrane in that the reagents generating the micro-phase separation were added to the feed solution. Then, the small decrease in acetone concentration or temperature decrease causes micro-phase separation to occur in the solution. The phase separation occurs in a short time at point on the membrane. The smaller pore size at both surfaces of membrane wall is due to increase in polymer concentration at the surfaces resulting from diffusion of acetone molecules from the casting solution into the external environment.

The pores in PVA hollow fiber membrane (by Kuraray Co.) is known to be prepared by extracting the soluble substance after spinning. The local stress distribution and local temperature distribution due to spinning in the radial 
direction may cause variation in the pore shape and mean pore size with $Z / d$. The PP hollow fiber membrane (by Mitsubishi Rayon Co.) is reported to be prepared by meltspinning, followed by cold drawing and heat treatment. The shape of the pore was the slit type and the mean pore size at both surfaces was smaller than that of the inside. Such heterogeneous shape may be due to those in drawing stress exerted on the hollow fiber.

A newly proposed method for evaluating pore characteristics, ASTS method, was found quite useful for analyzing the pore characteristics of hollow fiber membranes, whose pore diameters exceed $0.02 \mu \mathrm{m}$. Applying this method to various hollow fiber membranes, the dependence of pore characteristics on their location in the direction of membrane thickness was clarified and found closely related to compositional change in the cast solution in the thickness direction.

\section{REFERENCES}

1. K. Kamide and S. Manabe, "Ultrafiltration Membranes and Applications," A. R. Cooper, ed., Plenum Press, New York, N.Y., 1980, p 173.

2. U. Merin and M. Cheryan, J. Appl. Polym. Sci., 25, 2139 (1980).

3. H. A. Purz, C. Rosin, B. Philipp, K. J. Stiiller, and P. V. Zgglinicki, Acta Polymerica, 32, 726 (1981).

4. M. D. Vyas and I. C. Mody, Desalination, 42, 107 (1982).

5. K. Kamide and S. Manabe, "Role of Microphase Separation Phenomena in the Formation of Porous Polymeric membrane," from ACS Symposium Series, No. 269, "Material Science of Synthetic Membrane," The American Chemical Suciety, Washington, D.C. 1985.

6. S. Manabe, Y. Kamata, H. Iijima, and K. Kamide, Polym. J., 19, 391 (1987).

7. S. Manabe, Y. Shigemoto, and K. Kamide, Polym. J., 17, 775 (1985).

8. J. W. Cahn, Transaction of the Metallurgical Society of Aime, 242, 166 (1968).

9. K. Kamide and H. Iijima, unpublished results. 